\title{
Meta
}

Journal des traducteurs

Translators' Journal

\section{CABRERA, I., HÖRMANN, P., LOPEZ, E. y J. C. PALAZUELOS (1991): "Investigación en traducción : planteamientos y perspectivas", no especial de Taller de Letras, Pontificia Universidad Católica de Chilie, Facultad de Letras, Departamento de Traducción, 145 p.}

\section{Georges L. Bastin}

Volume 38, numéro 1, mars 1993

La traduction et l'interprétation dans le nord du Canada

Translation and Interpretation in Northen Canada

URI : https://id.erudit.org/iderudit/001986ar

DOI : https://doi.org/10.7202/001986ar

Aller au sommaire du numéro

Éditeur(s)

Les Presses de l'Université de Montréal

ISSN

0026-0452 (imprimé)

1492-1421 (numérique)

Découvrir la revue

Citer ce compte rendu

Bastin, G. L. (1993). Compte rendu de [CABRERA, I., HÖRMANN, P., LOPEZ, E. y J. C. PALAZUELOS (1991) : "Investigación en traducción : planteamientos y perspectivas", no especial de Taller de Letras, Pontificia Universidad Católica de Chilie, Facultad de Letras, Departamento de Traducción, 145 p.] Meta, 38(1), 129-130. https://doi.org/10.7202/001986ar 
CABRERA, I., HÖRMANN, P., LOPEZ, E. y J. C. PALAZUELOS (1991): «Investigación en traducción: planteamientos y perspectivas», $\mathrm{N}^{\circ}$ especial de Taller de Letras, Pontificia Universidad Católica de Chile, Facultad de Letras, Departamento de Traducción, $145 \mathrm{p}$.

Les ouvrages sur la traduction rédigés en espagnol étant assez rares, il convient de saluer la publication de cet ouvrage par le Département de traduction de l'Université catholique du Chili.

Né d'un état de la traduction dans quelques pays latino-américains dressé par les auteurs en 1990, l'ouvrage prétend combler certaines lacunes concernant la méthodologie, l'objet et l'évolution historique de la recherche traductologique. Trois chapitres s'y attachent avec rigueur et objectivité, mais avec une inégale réussite.

Le premier, Fondements et méthodes de la recherche en traductologie (Emilio López), pourrait, en toute rigueur, délester son titre du terme «traductologie» et le troquer, éventuellement, contre celui de «sciences sociales». En effet, l'auteur revisite les bases de la recherche scientifique universelle et tâche de les illustrer de manière fonctionnelle, en humaniste-traductologue. De toute évidence, il s'adresse à un public non averti, à des chercheurs en puissance qu'il vise à encourager et qu'il encourage à bien viser. Et ce n'est pas son moindre mérite que d'insister sur l'importance de cerner au plus près l'objet de la recherche. D. Seleskovitch ne répète-t-elle pas à ses thésards qu'une thèse est une tête d'épingle que l'on enfonce lentement mais profondément?

Emilio López offre ainsi de bien utiles réflexions au futur chercheur. S'il est vrai que son jargon aurait pu être moins lourd, il n'empêche que beaucoup d'universités hispanophones trouveront dans son travail les pistes à suivre pour mettre à profit le potentiel de recherche qui sommeille en elles.

Le second chapitre est intitulé $L$ 'objet de la traductologie et approches diverses $d u$ phénomène traductologique (Juan Carlos Palazuelos). L'auteur part de la définition aristotélicienne d' «activité» (propre, en puissance, réalisée) pour identifier les points de vue selon lesquels aborder la traductologie. Certes il revendique pour cette dernière un statut scientifique propre mais, il se laisse envoûter par les vertus de la «linguistique du texte» et de la «théorie du texte» comme cadre théorique pour répondre aux questions : «qu'estce qui est reproduit ?», «comment le reproduire ?» et «pourquoi le reproduire ?».

De nombreuses définitions de la traduction sont ensuite examinées, lesquelles appartiennent à plus de vingt auteurs en tout, (écrivains, linguistes et traductologues). Elles sont réparties en trois groupes: les «impressionnistes» (García Márquez, Gide, Dante, Cervantes, etc.), les «imprécises» (Cary, Catford, Vinay et Darbelnet, Seleskovitch, Delisle, Jakobson, Mounin, Nida, UNESCO, Königs, etc.) et les «doctrinales» (García Landa et Coseriu). Si la répartition est douteuse, en particulier le second groupe fourretout, la discussion des définitions n'est pas sans intérêt, dans la mesure où elle signale les vertus et les défauts de chacune. À noter qu'en dépit de certaines imprécisions, Coseriu est enfin sérieusement pris en compte.

Une «Récapitulation» reprend systématiquement les points de convergence et de divergence entre les multiples définitions étudiées, toujours par rapport aux trois questions posées précédemment.

Le «Commentaire» final retrace une petite histoire de la traductologie en trois moments : théorie linguistique, textuelle et pragmatique, et trois acteurs principaux : Mounin, García Landa et Königs. Il y a là matière à polémique, mais surtout à recherche, ce qui, en fin de compte, était l'objet du chapitre.

Le troisième chapitre, Évolution de la pensée traductologique au fil des temps (lleana Cabrera et Patricia Hörmann), tente un découpage nouveau de l'histoire de la traduction en trois étapes: normative, descriptive et explicative, lesquelles semblent répondre aux trois 
questions suivantes: 1) Quelle est la meilleure manière de traduire?, 2) Qu'est-ce que la traduction et qu'est-ce que traduire ? et 3) Quel est l'objet et quelles sont les méthodes de la traductologie? Sans prétendre à l'exhaustivité, les auteures répondent à ces questions sous la plume d'un choix d'auteurs. Ainsi, l'étape normative voit la primauté des «manières de bien traduire» grâce à saint Jérôme, Dolet et Luther. Bien que fixant le début de cette étape au IV ${ }^{\mathrm{e}}$ siècle après J.-C., les auteures ne négligent ni les commentaires de Horace ni ceux de Cicéron.

L'étape descriptive, au $\mathrm{XX}^{\mathrm{e}}$ siècle, est marquée par la description du processus traductif et la mise en relation de la traduction avec d'autres disciplines voisines. Juan Luis Vives et sa Typologie de la traduction selon les textes est le premier auteur analysé. Suivent Vinay et Darbelnet, Nida et Newmark, dont les positions théoriques sont constamment comparées à celles d'autres traductologues.

L'étape explicative est celle de la définition de l'objet et de la méthodologie propres de la traduction, avec pour représentants D. Seleskovitch et F. Königs.

Bref un panorama rondement mené, qui faisait cruellement défaut en espagnol.

L'ouvrage se termine sur une «Annexe», portant sur les Perspectives de la recherche traductologique en Amérique latine (Ileana Cabrera, Patricia Hörmann et Emilio López). Annexe peu judicieuse qui prétend mettre en parallèle la recherche traductologique en France, en Allemagne, aux États-Unis, au Canada et la recherche latino-américaine, de surcroît limitée au Cône sud. On pourrait s'indigner de ne pas y voir mentionnés les travaux de chercheurs mexicains, brésiliens et vénézuéliens, mais l'entreprise est ambitieuse et, quoi qu'il en soit, il y a des choses qu'il est préférable de ne pas comparer. C'est dommage, mais, si l'on se rappelle que ce diagnostic est à l'origine de l'ouvrage, les auteurs en sont pardonnés!

Faisons encore remarquer que cet ouvrage est le huitième (depuis 1981) à voir le jour au sein du Département de traduction de l'Université catholique du Chili. Longue vie à ses membres !

GEORGES L. BASTIN 